\title{
Elevated Fatty Acid Ethyl Esters in Meconium of Sheep Fetuses Exposed In Utero to Ethanol-A New Animal Model
}

\author{
YOAV LITTNER, TIMOTHY A. CUDD, MARY A. O'RIORDAN, ANDREW CWIK, AND CYNTHIA F. BEARER
}

\begin{abstract}
Department of Pediatrics [Y.L., M.A.O., A.C., C.F.B.], Department of Neurosciences and Environmental Health Sciences [C.F.B.], Case Western Reserve University and Rainbow Babies and Children's Hospital, Cleveland, Ohio 44106; Department of Veterinary Physiology and Pharmacology [T.A.C.], Texas A\&M University, College Station, Texas 77843
\end{abstract}

\begin{abstract}
Specific fatty acid ethyl esters (FAEE) in meconium of newborns have been shown to correlate with maternal ethanol exposure. An animal model is needed to assess the validity of this biomarker. We hypothesized that the pregnant/fetal sheep is a feasible animal model for validating FAEE as a biomarker of prenatal ethanol exposure. Nine pregnant ewes were treated during the third trimester with different i.v. ethanol doses. The control group consisted of 14 pregnant ewes exposed to similar volumes of saline. On gestational d 133, the fetuses were delivered and meconium samples removed. FAEEs were quantified by gas chromatography-flame ionization detection. FAEEs were found in both control and ethanol exposed fetuses. Ethyl oleate, ethyl linoleate, and ethyl arachidonate levels were significantly higher in the ethanol-exposed sheep. Ethyl oleate was the FAEE that correlated most strongly with alcohol ingestion during pregnancy and had the greatest area under the curve (0.94). Using a cut-off value of $131 \mathrm{ng} / \mathrm{g}$ ethyl oleate dry weight, sensitivity was $89 \%$ and specificity was $100 \%$. In conclusion, pregnant ewes are a feasible model for validating biomarkers of prenatal ethanol exposure. Ethyl oleate, ethyl linoleate, and ethyl arachidonate may be useful biomarkers of prenatal alcohol exposure. (Pediatr Res 63: 164-168, 2008)
\end{abstract}

A lcohol, one of the most frequently reported addictions in the U.S (1), is a known teratogen of the fetus. It is estimated that $1 \%$ of all U.S. newborns are affected by prenatal ethanol exposure (2), most of whom are not diagnosed at birth (3). Prevention is the best course of action, but, unfortunately, the results of current efforts are unsatisfactory. Early identification of an affected infant is important and may prevent secondary disabilities (4). Since maternal self-report is not a reliable diagnostic tool (5-7), the development of a biomarker for in utero alcohol exposure becomes a necessity.

To date, the most promising biomarkers are fatty acid ethyl esters (FAEE). FAEE are a stable product of nonoxidative ethanol metabolism and are formed by the conjugation of ethanol to endogenous FFA and fatty acyl-CoA. FAEE have been studied in different species (mouse, rat, sheep, guinea pig, and human) and can be detected in blood, hair, placenta,

Received May 9, 2007; accepted September 14, 2007

Correspondence: Yoav Littner, M.D., Division of Neonatology, Rainbow Babies and Children's Hospital, 11100 Euclid Avenue, Cleveland, OH 44106; e-mail: ylittner@hotmail.com

This research is supported by a grant from the National Institute on Alcohol and Alcohol Abuse, R03 AA12618 to CFB.

CFB currently holds a patent for the measurement of fatty acid ethyl ester in meconium. There are no business relationships based on this patent. and meconium of newborns exposed in utero to ethanol (Mac et al., Society for Pediatric Research, May 1-5, 1994, Seattle, WA) (8-12). Unlike blood, where FAEE have a short half-life (24 h after alcohol intake) (9), meconium and hair can accumulate FAEE for extended periods of time. Easy access and lack of invasiveness make meconium and hair attractive for the study of biomarkers.

Several human studies have shown that FAEE could serve as a reliable biomarker for prenatal alcohol use (11-15). However, the limitation of these studies is their dependence on maternal self-report. An objective, bias-free animal model for validating this biomarker is needed.

Several animal models have been used for investigating alcohol teratogenesis and/or validating biomarkers. Caprara et al. (10) used the guinea pig animal model to demonstrate that chronic exposure to alcohol during pregnancy resulted in increased levels of FAEE in both maternal and neonatal hair. Additionally, the pregnant ewe has been used as a model of fetal alcohol syndrome (16).

Here, we investigate whether the pregnant/fetal ewe is a feasible animal model for validating FAEE as a biomarker of prenatal ethanol exposure.

\section{METHODS}

Animals. This study was approved by the Texas A\&M University Institutional Animal Care and Use Committee and was subject to AAAALAC, PHS Policy on Humane Care and Use of Laboratory Animals. Suffolk ewes ranging in age from 2 to 6 y were bred to Suffolk rams. Estrus was induced by introducing vaginal progesterone implants (Eazi-Breed CIDR for sheep and goats, Pharmacia \& Upjohn, Auckland, NZ) and then removing them $12 \mathrm{~d}$ later. The following day, the ewes were mated with rams fitted with marking harnesses. The day that the ewes were marked by the rams was designated as gestational day (GD) 0 . Marked ewes were assessed ultrasonographically to confirm pregnancy and to determine the number of fetuses. Ewes were maintained in shaded outdoor pens with herdmates before mating until GD 90 . On GD 90, the ewes were moved indoors. A constant temperature of $22^{\circ} \mathrm{C}$ and a 12:12 light/dark cycle were maintained. All animals received $2 \mathrm{~kg} / \mathrm{d}$ of a "complete" ration (Sheep and Goat Pellet, Producers Cooperative Association, Bryan, TX).

Study design and ethanol dosing protocol. The human brain growth spurt peaks at the time of parturition, whereas the ovine brain growth spurt peaks approximately on GD 133 (17). Therefore, this study began on GD 109, the approximate beginning of the third trimester equivalent, and was terminated on GD 135 to correspond with the human in utero brain growth spurt.

The study consisted of five groups, including four ethanol treatment groups $(1.25,1.5,1.75$, and $2 \mathrm{~g} / \mathrm{kg})$ and a control group that received normal saline

Abbreviations: AUC, area under the curve; FAEE, fatty acid ethyl esters; GD, gestational day; ROC, receiver operating characteristic 
at a similar volume and infusion rate as the highest ethanol dose group. On GD 109, a catheter (16 G, 5.25-in Angiocath, Becton Dickinson, Sandy, UT) was placed percutaneously into the jugular vein. On GD 113, the ewes were anesthetized $(0.2 \mathrm{mg} / \mathrm{kg}$ diazepam, $4 \mathrm{mg} / \mathrm{kg}$ ketamine, $0.5-0.25 \%$ isoflurane $)$ and a polyvinyl chloride catheter was placed in the vena cava via insertion into the femoral vein. Infusion solutions were delivered by peristaltic pump (Masterflex, model 7014-20, Cole Parmer, Niles, IL) through a $0.2-\mu \mathrm{m}$ bacteriostatic filter. Pumps were calibrated before each infusion. Ethanol infusions were $40 \% \mathrm{wt} / \mathrm{vol}$ in sterile saline administered over $1 \mathrm{~h}$. The ethanol dosing regimen was designed to mimic a common human binge pattern of drinking; ewes received ethanol on three consecutive days followed by $4 \mathrm{~d}$ without ethanol from GD 109 to 132 of gestation. The last dose of ethanol was given on GD 132. Nine ewes were entered into the treatment groups and 14 ewes were in the control group.

Meconium collection and preparation. On GD 133, approximately $24 \mathrm{~h}$ after the last dose of ethanol, the ewes were euthanized by an overdose of sodium pentobarbital $(75 \mathrm{mg} / \mathrm{kg}$ ). The fetus was then removed from the uterus and weighed. The large intestine of the fetus was dissected intact from the peritoneal cavity and quickly frozen in liquid nitrogen. At the time of analysis, the specimen was weighed and total length measured. The intestine was opened longitudinally, and all meconium removed, weighed, and analyzed for FAEE.

$\boldsymbol{F A E E}$ analysis. Isolation of FAEE from meconium was performed as previously described (18). The total amount of meconium (removed from one intestine) was divided into multiple aliquots of $1 \mathrm{~g}$ wet weight. FAEE were isolated and quantified by gas chromatography-flame ionization detection. For each animal, the mean value of each FAEE was calculated by averaging the FAEE ng/g measured values of all 1-g aliquot. Personnel performing FAEE analysis were blinded to exposure conditions.

Statistical analysis. Analyses were carried out to compare meconium weight and FAEE levels between the ethanol exposed sheep and controls. For these comparisons, we used $t$ test for variables with normal distribution and the nonparametric Wilcoxon test when the distribution was not normal. To test the correlation between FAEE levels and dosage of ethanol exposure, we conducted a linear regression analysis. A receiver operating characteristic (ROC) analysis was performed to assess the ability of FAEE to differentiate between ethanol-exposed sheep and controls. ROC curves of FAEE were plotted and the area under the curve (AUC) calculated. Using the ROC curves, the cut-off values that provide both the highest sensitivity and the highest specificity were determined and sensitivity rate, specificity rate, and positive and negative predictive values were calculated.

The Wilcoxon signed-rank test was used to compare between FAEE concentrations in the first 1-g aliquot (proximal) and last 1-g aliquot (distal) colon segments of each animal. A qualitative comparison between our finding and a previous human study was conducted without formal test of significance. The SAS software version 8.1 was used for analyses (SAS Institute, Carey, NC). $p$ Values $<0.05$ were considered significant.

\section{RESULTS}

The amount of meconium in ovine fetuses did not differ between the ethanol exposed group and controls (11.6 versus $10.6 \mathrm{~g}$, respectively, $p=0.54$ ). In addition, we did not find any correlation between the amount of meconium and ethanol dosage.

FAEE were detected in both control and ethanol exposed sheep (Table 1). Control sheep had predominantly ethyl palmitoleate $>$ ethyl linoleate $>$ ethyl myristate. The predominant
FAEE differed in ethanol exposed sheep and were ethyl palmitoleate $>$ ethyl oleate $>$ ethyl linoleate. Of interest, ethyl arachidonate was found only in the meconium of ethanol exposed animals.

The mean concentrations of some FAEE were significantly higher in ethanol exposed animals compared with control animals. As shown in Table 1, the mean concentrations of ethyl oleate, ethyl linoleate, and ethyl arachidonate were significantly higher than those of control animals. There was a trend toward significance with ethyl palmitate and ethyl linolenate. The predominant FAEE in both control and ethanol exposed sheep, ethyl palmitoleate, was not significantly different between the two groups.

To determine whether the sum of all FAEE was significantly different between control and treated groups, we summed the mean values of FAEE for each animal (Table 1). This total FAEE concentration had higher levels in ethanol exposed fetuses compared with control (1252 and $827 \mathrm{ng} / \mathrm{g}$, respectively). However, this difference was not statistically significant $(p=0.115)$. When the mean of the concentrations of three FAEEs (ethyl oleate, ethyl linoleate, and ethyl arachidonate) was summed, a significant association between ethanol exposure and higher levels of FAEE was seen (306 $\mathrm{ng} / \mathrm{g}$ in controls versus $495 \mathrm{ng} / \mathrm{g}$ in ethanol exposed, $p=$ 0.002) (Table 1).

The relationships between alcohol dosage and FAEE concentrations were examined using a linear regression analysis. We did not find any correlation between FAEE levels and ethanol dosage. To address whether meconium FAEE varied according to the time of exposure, we compared the FAEE concentration in the first (proximal) $1-\mathrm{g}$ aliquot and last (distal) 1-g aliquot. No significant difference between the first and last aliquot was seen either in control or in ethanol-treated animals.

To assess the efficiency of FAEE as a biomarker for ethanol exposure we conducted a ROC analysis (Table 2). For each FAEE, a ROC curve was plotted and the AUC calculated. Figure 1 shows a representative ROC curves for the three most useful FAEE. Of all FAEE, ethyl oleate was the best indicator of alcohol exposure with an AUC of 0.94 (95\% confidence interval, $0.83-1.00$ ). At a cut-off value of $131 \mathrm{ng} / \mathrm{g}$ wet weight ethyl oleate, sensitivity was $89 \%$, specificity was $100 \%$, positive predictive value was 1.00 , and negative predictive value was 0.93. Other FAEE showing significant difference were ethyl arachidonate and ethyl linoleate with AUC of 0.78 and

Table 1. The mean concentration of FAEE in the meconium of control and ethanol exposed sheep

\begin{tabular}{lccc}
\hline \multicolumn{1}{c}{ FAEE } & Control $(\mathrm{ng} / \mathrm{g})$, median (range) & Ethanol exposed (ng/g), median (range) & $p$ Value \\
\hline Ethyl myristate 14:0 & $110(0-699)$ & $136(50-336)$ & $0.77 *$ \\
Ethyl palmitate 16:0 & $87(18-366)$ & $33(20-776)$ & $0.09^{*}$ \\
Ethyl palmitoleate 16:1 & $307(160-716)$ & $298(28-1045)$ & $0.81 \dagger$ \\
Ethyl oleate 18:1 & $69(0-131)$ & $293(117-878)$ & $<0.001^{*}$ \\
Ethyl linoleate 18:2 & $215(61-441)$ & $8.2(0-626)$ & $0.05^{*}$ \\
Ethyl linolenate 18:3 & $0(0-57)$ & $6.6(0-224)$ & $0.07 *$ \\
Ethyl arachidonate 20:4 & $0(0)$ & $1252(303-3873)$ & $0.002^{*}$ \\
Sum of total FAEEs & $827(458-1567)$ & $495(145-1703)$ & $0.115^{*}$ \\
Sum of 3 FAEEs (18:1+18:2+20:4) & $306(81-544)$ & & $0.002^{*}$ \\
\hline
\end{tabular}

\footnotetext{
$*$ Wilcoxon test; $\uparrow t$ test.
} 
Table 2. ROC analysis

\begin{tabular}{lcccrrr}
\hline \multicolumn{1}{c}{ FAEE } & AUC $(\%)$ & Cut-off* & Sensitivity $(\%)$ & Specificity $(\%)$ & PPV $(\%)$ & NPV $(\%)$ \\
\hline Ethyl myristate 14:0 & 54 & 116 & 56 & 57 & 45 \\
Ethyl palmitate 16:0 & 72 & 98 & 67 & 86 & 67 \\
Ethyl palmitoleate 16:1 & 51 & 339 & 56 & 64 & 75 \\
Ethyl oleate 18:1 & 94 & 131 & 89 & 50 & 69 \\
Ethyl linoleate 18:2 & 75 & 247 & 78 & 79 & 700 \\
Ethyl linolenate 18:3 & 71 & 0 & 67 & 70 & 67 \\
Ethyl arachidonate 20:4 & 78 & 0 & 56 & 100 & 79 \\
Sum of three FAEEs (18:1+18:2+20:4) & 88 & 376 & 93 & 89 & 100 \\
\hline
\end{tabular}

AUC, area under the curve; PPV, positive predictive value; NPV, negative predictive value.

$* \mathrm{ng} / \mathrm{g}$.

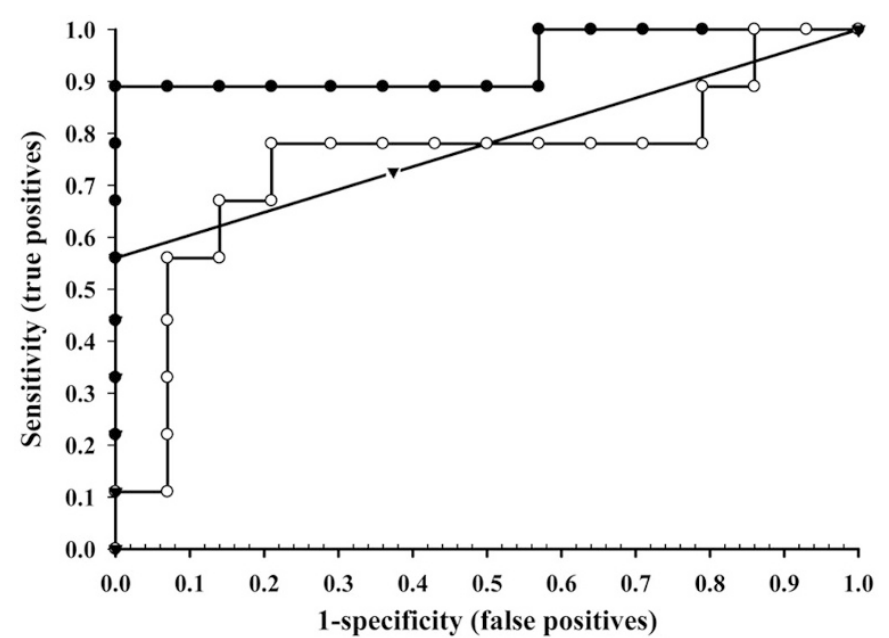

Figure 1. ROC curve assessing the sensitivity and specificity of ethyl oleate, ethyl linoleate, and ethyl arachidonate concentrations in meconium for identifying prenatal ethanol exposure. Ethyl oleate (18:1) (O); ethyl linoleate $(18: 2)(\bigcirc)$; and ethyl arachidonate $(20: 4)(\boldsymbol{\nabla})$.

0.75 , respectively. Ethyl palmitate and ethyl linolenate had AUC of 0.72 and 0.71 , respectively, but did not reach statistical significance. A ROC analysis of the cumulative three FAEE concentrations generated an AUC of 0.88 with a $95 \%$ confidence interval of $0.71-1.00$. At a cut-off value of 376 $\mathrm{ng} / \mathrm{g}$, sensitivity was $89 \%$, specificity was $93 \%$, positive predictive value was 0.89 , and negative predictive value was 0.93 .

An essential step in validating any animal model is a comparison to human studies. The comparison between our findings and our previously reported Cleveland population (abstainers and nonabstainers groups) (13) (Fig. 2) yielded several observations. In the control groups, the profile of the FAEE was different between sheep and human. The main difference was in the predominant FAEE. In sheep controls, the predominant FAEE were ethyl palmitoleate, ethyl linolenate, and ethyl myristate whereas in human controls they were ethyl palmitoleate, ethyl oleate, and ethyl linolenate. Beside these differences, we recognized a resemblance between the levels of most FAEE among sheep and human controls. Notably, ethyl arachidonate was not found in the meconium of control sheep.

In the groups exposed prenatally to ethanol, both sheep and humans demonstrated a general raise in FAEE levels compared with controls. Moreover, the same FAEE become predominant in human and sheep (ethyl palmitoleate, ethyl oleate, and ethyl linolenate). As opposed to the human study where six of seven

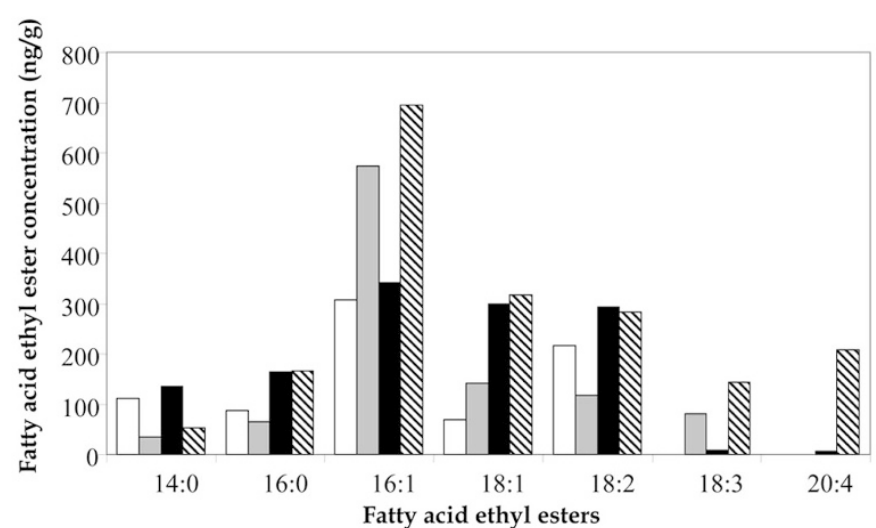

Figure 2. Median fatty acid ethyl ester levels (ng/g) in meconium of control and alcohol exposed sheep and humans. Sheep controls (open bars), human controls (gray bars), ethanol exposed sheep (black bars), ethanol-exposed humans (hatched bars). The data of human groups reflects Cleveland's abstainers and nonabstainers and was modified with permission from Bearer CF et al. J Pediatr 146:824-830. Copyright (C) 2005 Elsevier Inc.

FAEE were significantly different between nonabstainers and abstainers groups (13), in sheep, only ethyl oleate, ethyl linoleate, and ethyl arachidonate levels were significantly higher in the ethanol exposed group.

Lastly, using ROC analysis, both studies found ethyl oleate, ethyl linoleate, and ethyl arachidonate to be the best indicators of prenatal ethanol exposure.

\section{DISCUSSION}

In the last decade, our understanding of ethanol teratogenicity has substantially expanded. Nevertheless, our ability to screen newborns for prenatal ethanol exposure is still lacking. Except for cases that present with obvious features of fetal alcohol syndrome, most babies exposed in utero to ethanol are neither dysmorphic nor present with any characteristics that help with the diagnosis. As a result, the majority of babies are not diagnosed until late childhood $(3,19)$. It became clear that maternal self-report is not sensitive or reliable (5) and alternative ways for screening are needed.

FAEE is one of the biomarkers that has emerged because of ongoing research. Both meconium and neonatal hair are good materials for collecting and measuring FAEE accumulated during fetal life. Studies of pregnant women have found a correlation between elevated FAEE levels in meconium and alcohol consumption $(11-13,15,20)$. However, further valida- 
tion of this biomarker requires confirmation with an animal model that eliminates the dependence on maternal self-report.

In this study, we used the sheep model for validating FAEE as a biomarker of prenatal ethanol exposure. We selected the sheep fetus due to its similarity to the human fetus. The sheep fetus has a relatively long gestation (147 d). Fetal size is equivalent to that of humans and has a similar prenatal high-velocity brain growth as in humans (16).

Using this model, we demonstrated that prenatal ethanol exposure resulted in higher FAEE levels compared with control. Sheep ethyl oleate was the FAEE with the most significant difference between ethanol exposed and control groups. Other FAEE, such as ethyl linoleate and ethyl arachidonate, also had significantly higher levels in the ethanol exposed sheep. However, their ability to predict ethanol exposure [AUC of 0.78 and 0.75 respectively (Table 2)] was less than that of ethyl oleate.

The resemblance of our findings to those of a recent human study (13) is of great importance. Both sheep and human FAEE levels were the same order of magnitude. In addition, both studies reported the same three FAEE as the best indicators of ethanol exposure. These observations not only support our hypothesis but also authenticate the results of the human study.

Other reported observations have also shown a significant association between elevated FAEE in meconium and in utero alcohol exposure $(11,12,15,20,21)$. However, these studies vary in the types of FAEE found to be highly indicative of ethanol exposure. The FAEE identified in these studies included ethyl linoleate $(11,15,21)$, ethyl oleate $(12,21)$, ethyl arachidonate (15), ethyl laurate, ethyl palmitate, and ethyl stearate (20). The results from existing animal research are not homogenous either. Brien et al. (22) reported a 5-fold higher concentrations of ethyl laurate, ethyl palmitate, ethyl stearate, and ethyl oleate in meconium of term fetal guinea pigs exposed prenatally to ethanol. A different model using Guinea pig hair found an association between ethyl oleate and ethanol exposure (23). There is no evident explanation for the differences between the various studies and our findings. The different populations studied, maternal diet (24), and genetic polymorphism may play an important role in influencing FAEE composition.

Our findings of elevated ethyl arachidonate levels in ethanol exposed animal are intriguing for several reasons. First, ethanol reduces blood linoleic acid levels (the precursor of arachidonate) and blocks conversion of linoleic acid and gamma-linolenic acid to arachidonic acid $(25,26)$. This casts doubt on the source of elevated ethyl arachidonate levels in ethanol exposed animals. Probably, ethanol's ability to increase phospholipase-A2 and phospholipase-C activity (27) causes a release of arachidonic acid from membrane phospholipids that later on is converted to ethyl arachidonate. Second, none of the specimens from the control group contained ethyl arachidonate. A study by Moore et al. (21) supports our findings, whereas others have shown detectable levels of ethyl arachidonate in controls $(13,15)$. Refaai et al. (28) reported the presence of ethyl arachidonate only in tissue of individuals with detectable blood ethanol at the time of autopsy. These conflicting reports raise important questions. Were newborns in the human control group exposed prenatally to alcohol? Can we be absolutely sure of the drinking history of the mothers? Regardless of the answer, if our observation is true, any detection of ethyl arachidonate in meconium will be exceptionally specific for ethanol exposure. We believe that a combination of ethyl arachidonate and ethyl oleate will provide a highly sensitive and specific screening test for ethanol exposure.

Interestingly, fetuses from the control group had significant amounts of FAEE in the meconium. It is evident from preliminary human studies that FAEE can be found in the meconium of newborns without prenatal ethanol exposure $(12,13,15,29)$. Other animal studies also found small amounts of FAEE in hair of neonatal guinea pigs that were not exposed prenatally to ethanol (10). As for the human studies, one can always claim that maternal history is not accurate and some of the babies in the abstaining group were actually exposed in utero to ethanol. Also, small quantities of ethanol may be present in certain medication, maternal diet [e.g. olive oil (30)] and food additives. Lastly, it is known that small amounts of ethanol are a normal by-product of metabolism in the human gut (31). Most likely, FAEE found in controls are a result of both ingestion and/or endogenous production of ethanol.

Although animals in the ethanol treatment group showed an association between ethanol dose and FAEE levels (data not shown), it did not reach statistical significance. Probably, the small sample size of this study accounts for this lack of statistical significance. In addition, no difference in regional distribution of FAEE was found. However, this study was not designed to determine regional distribution, so this result should be interpreted cautiously.

A number of studies suggested the use of combined FAEE concentrations as a better biomarker of ethanol exposure $(10,22)$. We conducted a similar analysis using the sum of FAEE concentrations. Ethanol-exposed fetuses did have higher levels of total FAEE, but the difference was not statistically significant. When a combination of the three FAEE with the most significant differences between the groups was used, significantly higher levels of cumulative FAEE were noticed in the ethanol-exposed group $(p=0.02)$. Yet, the use of combined FAEE did not have any advantage over ethyl oleate as a biomarker of alcohol consumption (AUC of 0.88 versus 0.94 , respectively).

Our study is not without limitations. This pilot study included a small number of animals that might not reflect the true range and levels of FAEE in meconium. Moreover, the small sample size makes it harder to find a true correlation between different ethanol dosages and FAEE levels even if it exists, leading to type II error. In addition, the method we used for ethanol exposure (parenteral infusion) is different from the way ethanol is consumed by humans. The "physiologic" absorption of ethanol in humans may result in a different pattern of blood alcohol levels and may affect FAEE levels in a different way compared with i.v. infusion of alcohol.

Given that humans and sheep do not consume the same diet, it is possible that fatty acids composition will differ between the species. As fatty acids are the source of FAEE, it was essential to compare between the two species. Surprisingly, 
plasma fatty acids composition in pregnant sheep and pregnant women did not differ significantly $(32,33)$. Our ruminant animal model also has a different type of digestion than humans. Ruminant animals are capable of producing small amounts of alcohol from fermentation of glycerol (34), which could explain the presence of FAEE in the meconium of our controls. Nevertheless, this is not different from human's endogenous production of ethanol and should not impede future use of ruminant animals as an animal model for ethanol exposure.

The ability to collect all of the meconium is feasible with an animal model, but more difficult in a clinical setting, especially when some infants pass their first meconium before delivery. When all the samples are collected, the chance of a bias is decreased. Thus, a random sample of meconium is most probably a practical way for screening but may carry the risk of a bias given that FAEE can accumulate unevenly in meconium. However, our preliminary results do not support an uneven distribution of FAEE within meconium.

To our best knowledge, this is the first study using the sheep model to test the association between FAEE in meconium and ethanol exposure. It is an important step toward our ability to validate the biomarker in an objective way. Furthermore, it partially supports the results found in human studies making the probability for a bias in these studies less likely.

We conclude that sheep are a reliable and feasible model for validating biomarkers of prenatal ethanol exposure. Specific FAEE in meconium, in particular ethyl oleate, ethyl linoleate, and ethyl arachidonate, may provide an accurate indicator of maternal alcohol use during pregnancy. Further studies with larger number of animals are needed and may provide the ability to determine the pattern, amount, and timing of ethanol exposure according to levels and profile of specific FAEE in meconium.

\section{REFERENCES}

1. Abel EL, Sokol RJ 1991 A revised conservative estimate of the incidence of FAS and its economic impact. Alcohol Clin Exp Res 15:514-524

2. Sampson PD, Streissguth AP, Bookstein FL, Little RE, Clarren SK, Dehaene P, Hanson JW, Graham JM 1997 Incidence of fetal alcohol syndrome and prevalence of alcohol-related neurodevelopmental disorder. Teratology 56:317-326

3. Little BB, Snell LM, Rosenfeld CR, Gilstrap LC 3rd, Gant NF 1990 Failure to recognize fetal alcohol syndrome in newborn infants. Am J Dis Child 144:11421146

4. Streissguth AP, Bookstein FL, Barr HM, Sampson PD, O’Malley K, Young JK 2004 Risk factors for adverse life outcomes in fetal alcohol syndrome and fetal alcohol effects. J Dev Behav Pediatr 25:228-238

5. Russell M, Martier SS, Sokol RJ, Mudar P, Jacobson S, Jacobson J 1996 Detecting risk drinking during pregnancy: a comparison of four screening questionnaires. Am J Public Health 86:1435-1439

6. Stoler JM, Huntington KS, Peterson CM, Peterson KP, Daniel P, Aboagye KK, Lieberman E, Ryan L, Holmes LB 1998 The prenatal detection of significant alcohol exposure with maternal blood markers. J Pediatr 133:346-352

7. Walsh RA, Redman S, Adamson L 1996 The accuracy of self-report of smoking status in pregnant women. Addict Behav 21:675-679
8. Bearer CF, Gould S, Emerson R, Kinnunen P, Cook CS 1992 Fetal alcohol syndrome and fatty acid ethyl esters. Pediatr Res 31:492-495

9. Soderberg BL, Sicinska ET, Blodget E, Cluette-Brown JE, Suter PM, Schuppisser T, Vetter W, Laposata M 1999 Preanalytical variables affecting the quantification of fatty acid ethyl esters in plasma and serum samples. Clin Chem 45:2183-2190

10. Caprara DL, Brien JF, Iqbal U, Reynolds JN, Klein J, Koren G 2005 A guinea pig model for the identification of in utero alcohol exposure using fatty acid ethyl esters in neonatal hair. Pediatr Res 58:1158-1163

11. Bearer CF, Lee S, Salvator AE, Minnes S, Swick A, Yamashita T, Singer LT 1999 Ethyl linoleate in meconium: a biomarker for prenatal ethanol exposure. Alcohol Clin Exp Res 23:487-493

12. Bearer CF, Jacobson JL, Jacobson SW, Barr D, Croxford J, Molteno CD, Viljoen DL, Marais AS, Chiodo LM, Cwik AS 2003 Validation of a new biomarker of fetal exposure to alcohol. J Pediatr 143:463-469

13. Bearer CF, Santiago LM, O'Riordan MA, Buck K, Lee SC, Singer LT 2005 Fatty acid ethyl esters: quantitative biomarkers for maternal alcohol consumption. J Pediatr 146:824-830

14. Moore C, Jones J, Lewis D, Buchi K 2003 Prevalence of fatty acid ethyl esters in meconium specimens. Clin Chem 49:133-136

15. Ostrea EM, Hernandez JD, Bielawski DM, Kan JM, Leonardo GM, Abela MB, Church MW, Hannigan JH, Janisse JJ, Ager JW, Sokol RJ 2006 Fatty acid ethyl esters in meconium: are they biomarkers of fetal alcohol exposure and effect? Alcohol Clin Exp Res 30:1152-1159

16. Cudd TA 2005 Animal model systems for the study of alcohol teratology. Exp Biol Med (Maywood) 230:389-393

17. Dobbing J, Sands J 1979 Comparative aspects of the brain growth spurt. Early Hum Dev 3:79-83

18. Bearer CF 1999 Detection of Teratogen Exposure. U.S. Patent Documents. Case Western Reserve University, Cleveland, OH

19. Abel EL 1996 Fetal Alcohol Syndrome: From Mechanism to Prevention. CRC Press, Inc., Boca Raton, FL

20. Klein J, Karaskov T, Koren G 1999 Fatty acid ethyl esters: a novel biologic marker for heavy in utero ethanol exposure: a case report. Ther Drug Monit 21:644-646

21. Moore CM, Lewis D 2001 Fatty acid ethyl esters in meconium: biomarkers for the detection of alcohol exposure in neonates. Clin Chim Acta 312:235-237

22. Brien JF, Chan D, Green CR, Iqbal U, Gareri J, Kobus SM, McLaughlin BE, Klein J, Rao C, Reynolds JN, Bocking AD, Koren G 2006 Chronic prenatal ethanol exposure and increased concentration of fatty acid ethyl esters in meconium of term fetal guinea pig. Ther Drug Monit 28:345-350

23. Kulaga V, Caprara D, Iqbal U, Kapur B, Klein J, Reynolds J, Brien J, Koren G 2006 Fatty acid ethyl esters (FAEE); comparative accumulation in human and guinea pig hair as a biomarker for prenatal alcohol exposure. Alcohol Alcohol 41:534-539

24. Stark KD, Beblo S, Murthy M, Whitty JE, Buda-Abela M, Janisse J, Rockett H, Martier SS, Sokol RJ, Hannigan JH, Salem NJ 2005 Alcohol consumption in pregnant, black women is associated with decreased plasma and erythrocyte docosahexaenoic acid. Alcohol Clin Exp Res 29:130-140

25. Horrobin DF 1987 Essential fatty acids, prostaglandins, and alcoholism: an overview. Alcohol Clin Exp Res 11:2-9

26. Valles SL, Blanco AM, Pascual M, Guerri C 2004 Chronic ethanol treatment enhances inflammatory mediators and cell death in the brain and in astrocytes. Brain Pathol 14:365-371

27. Natsuki R, Yamaguchi T 1996 Effects of ethanol on phospholipases in the mouse brain, heart and liver. Biol Pharm Bull 19:525-529

28. Refaai MA, Nguyen PN, Steffensen TS, Evans RJ, Cluette-Brown JE, Laposata M 2002 Liver and adipose tissue fatty acid ethyl esters obtained at autopsy are postmortem markers for premortem ethanol intake. Clin Chem 48:77-83

29. Chan D, Bar-Oz B, Pellerin B, Paciorek C, Klein J, Kapur B, Farine D, Koren G 2003 Population baseline of meconium fatty acid ethyl esters among infants of nondrinking women in Jerusalem and Toronto. Ther Drug Monit 25:271-278

30. Perez-Camino MC, Moreda W, Mateos R, Cert A 2002 Determination of esters of fatty acids with low molecular weight alcohols in olive oils. J Agric Food Chem 50:4721-4725

31. Ostrovsky YM, Pronko PS, Shishkin SN, Kolesnikov VB, Volynets SI 1989 An attempt to evaluate diagnostic and prognostic significance of blood endogenous ethanol in alcoholics and their relatives. Alcohol 6:97-102

32. Hendrickse W, Stammers JP, Hull D 1985 The transfer of free fatty acids across the human placenta. Br J Obstet Gynaecol 92:945-952

33. Elphick MC, Hull D, Broughton PF 1979 The transfer of fatty acids across the sheep placenta. J Dev Physiol 1:31-45

34. Jarvis GN, Moore ER, Thiele JH 1997 Formate and ethanol are the major products of glycerol fermentation produced by a Klebsiella planticola strain isolated from red deer. J Appl Microbiol 83:164-174 